\title{
La dualidad presente en la educación profesional de Brasil y Argentina: un análisis comparado de los decretos y leyes educativas
}

The duality present in professional education in Brazil and Argentina: a comparative analysis of educational decrees and laws

A dualidade presente na educação profissional do Brasil e da Argentina: uma análise comparada dos decretos e leis educacionais

\section{Resumen}

En este artículo analizamos los decretos y leyes que retratan la Educación Profesional en la segunda mitad del siglo XIX y en el transcurso del siglo XX, en Brasil y Argentina. Tomamos como punto de partida el proceso de expansión de la escuela en esos espacios, en razón de coadunarse al movimiento internacional de escolarización de las masas populares ocurrido en el siglo XIX, con vistas a la formación de mano de obra para el trabajo, en alineamiento con los ideales liberales difundidos en América Latina. Esos ideales se difunden a través de leyes que refuerzan la dualidad educacional y contribuyen al aumento de las desigualdades sociales, siendo la escuela uno de los espacios fundamentales de disputas políticas y sociales. Seleccionamos, para ese fin, fuentes documentales y bibliográficas referentes a reglamentos que tratan sobre educación profesional en Brasil y Argentina. Utilizamos ese recorte hermanado de espacio y tiempo, buscando percibir por intermédio de la metodología de la Historia Comparada las diferencias y semejanzas, pero también las singularidades, para así pensar en las influencias compartidas entre esas dos realidades. Concluyendo que, esos países se acercan en lo que respecta a sus modelos educativos orientados al trabajo y están fuertemente dirigidos por los intereses de las elites y de los organismos internacionales en el proceso de construcción de sus leyes y reformas educativas, que no siempre son sinónimos de progreso e inclusión.

Palabras clave: Historia de la educación; Educación comparada; Educación profesional; Legislación educacional.

\begin{abstract}
In this article we analyze the decrees and laws that portray Professional Education in the second half of the 19th century and throughout the 20th century, in Brazil and Argentina. We took as a starting point the process of school expansion in these spaces, as it is in line with the international movement of schooling of the popular masses that took place in the nineteenth century, intending to train labor for work, in line with ideals liberals spread in Latin America. These ideals are disseminated through laws that reinforce the educational duality and contribute to the increase in social inequalities, with the school being one of the fundamental spaces for political and social disputes. For this purpose, we selected documental and bibliographical sources referring to rules that deal with professional education in Brazil and Argentina. We use this twinned cut of space and time, trying to understand, through the methodology of Comparative History, the differences and similarities, but also the singularities, so that we can think about the shared influences between these two realities. In conclusion, these countries are closer in terms of their educational models aimed at work and are strongly guided by the interests of elites and international organizations in the process of building their laws and educational reforms, which are not always synonymous of progress and inclusion.
\end{abstract} Keywords: Education history; Comparative education; Professional education; Educational legislation. 


\begin{abstract}
Resumo
Neste artigo analisamos os decretos e leis que retratam a Educação Profissional na segunda metade do século XIX e no decurso do século XX, no Brasil e na Argentina. Tomamos como ponto de partida o processo de expansão da escola nesses espaços, em razão de coadunar-se ao movimento internacional de escolarização das massas populares ocorrido no século XIX, com vistas à formação de mão de obra para o trabalho, em alinhamento com os ideais liberais difundidos na América Latina. Esses ideais são disseminados através de leis que reforçam a dualidade educacional e contribuem para aumento das desigualdades sociais, sendo a escola um dos espaços fundamentais de disputas políticas e sociais. Selecionamos, para esse fim, fontes documentais e bibliográficas referentes a regramentos que tratam sobre educação profissional no Brasil e na Argentina. Utilizamos esse recorte geminado de espaço e tempo, procurando perceber por intermédio da metodologia da História Comparada às diferenças e semelhanças, mas também as singularidades, para assim pensarmos nas influências compartilhadas entre essas duas realidades. Concluindo-se que, esses países aproximam-se no que diz respeito aos seus modelos educacionais voltados para o trabalho e são fortemente direcionados pelos interesses das elites e dos organismos internacionais no processo de construção das suas leis e reformas educacionais, que nem sempre são sinônimos de progresso e inclusão.
\end{abstract}

Palavras-chave: História da educação; Educação comparada; Educação profissional; Legislação educacional.

\title{
1. Introducción
}

A principios del inicio del siglo XIX en Brasil y Argentina la institucionalización de la instrucción pública dirigida para clases populares no se presentaba como una cuestión central en las decisiones políticas gubernamentales. Solamente a mediados del siglo XIX, con la expansión de los ideales de construcción de nación1 y difusión de los ideales liberales, que se construye tanto en el escenario brasileño como argentino el establecimiento de la necesidad de una expansión de la escuela para las clases populares.

La historia de la educación tanto en Brasil como en Argentina, se conformó en sus bases de institucionalización en el sentido de atender claramente a las élites políticas y al trabajo intelectual, dejando el trabajo manual reservado a las clases populares. Según Alejandro de la Fuente, et al (2018, p. 86), en parte como resultado de las prácticas raciales del período colonial, los indicadores de cualificación profesional de los negros en las nuevas naciones independientes suramericanas no se muestran muy diferentes. En Argentina, el censo de Buenos Aires de 1827 encontró los negros y pardos libres trabajando principalmente en actividades manuales y casi completamente ausentes de las filas de propietarios de inmuebles, comerciantes o profesionales liberales.

Tratándose de Brasil, Luiz Antônio Cunha (2005) aclara que el trabajo manual, entendido como trabajo subalterno, fue destinado a los ex-esclavizados y a los pobres y abandonados, los denominados "desvalidos de la suerte" mientras que el trabajo intelectual estaba reservado a los hombres libres ricos. Esta desigualdad y diferenciación social era reproducida directamente en la enseñanza destinada a esas clases.

Esas relaciones establecidas en el ámbito de la escolarización y del trabajo pueden ser claramente percibidas cuando recurrimos a las leyes y decretos producidos en esos países. Esa serie de reglamentos representaban el diseño que los grupos económicos que dominaban la política, pretendían establecer como proyecto de nación.

A partir de los dispositivos legales, que hacían referencia a la Educación Profesional en la segunda mitad del siglo XIX y en el transcurso del siglo XX, en Brasil y en Argentina buscamos entender las influencias de los ideales liberales en la organización escolar dirigida al trabajo, y, cómo esas instancias han contribuido de manera significativa para el aumento de las desigualdades sociales y para la dualidad de la enseñanza, teniendo la escuela como un espacio importante para la difusión de ideales liberales.

Para contestar esas cuestiones utilizamos los presupuestos de la Historia Comparada de la educación, emprendiendo

\footnotetext{
1 "La constitución de la forma escolar de educación procesada en el siglo XIX fue ejecutada en medio a disputas y tensiones, asociada a los proyectos de nación y la formación de los Estados Nacionales. El oitocentos es un período fértil de debates sobre la construcción de las identidades nacionales, iniciativas y prácticas educativas". El tema. (Gondra \& Schueler, 2008, p. 19) [...] "El tema del nacionalismo, a finales del siglo XIX y principios del XX, fue un componente de la pauta de interés de muchos sectores sociales que involucran aspectos como la educación y las relaciones internacionales. La nación sólo podría definirse como tal porque existían naciones, que cumplían con los estándares de la civilización occidental moderna, normalizados a partir de los países centrales" (Kuhlmann Júnior, 2015, pp. 40-41)
} 
un análisis de las diferencias, semejanzas, de las analogías, de las identificaciones, de los intercambios y también de las singularidades presentes entre esos dos países. Sin embargo, sentimos la necesidad también de pensar cómo esas ideas resuenan en el contexto socio-político latinoamericano.

Nuestro análisis comparado parte del presupuesto que en el caso de la educación en América Latina y en otros países del Tercer Mundo, las políticas y leyes educacionales, en lo que se refiere a una parte significativa del siglo XX, se derivan de acuerdos y de la intervención de organismos internacionales. (Ciavatta, 2009) Por lo tanto, las aproximaciones entre Brasil y Argentina son relevantes, teniendo en cuenta que sus posiciones socioeconómicas de periferia del capital, deja marcas en lo que concierne a los modelos de educación para el trabajo de la masa trabajadora, es decir, de la población explotada por el capital y el capitalismo. (Ciavatta, et al, 2019)

Para este estudio, fue necesario pensar particularmente los países de la América Latina, en el ámbito de la formación para el trabajo, teniendo en cuenta que, al realizar comparaciones con países desarrollados, terminan por mantenerse en el lugar común deseado por estas naciones, o sea, en su posición de dominados. Y, en este proceso de subalternización, pierden el referencial de crítica a las imposiciones históricamente puestas por estos países, reforzadas por los organismos internacionales. Al dejar de mirar a sus vecinos y a su espacio y contexto interno, desenfocan la visión y ven de manera miope sus problemas socio-políticos e insisten en buscar la solución donde justamente son generados. Por eso, la necesidad de comprensión de esas dos realidades, dejando aparte las comparaciones con las naciones llamadas del capitalismo central.

Hay que considerar los intercambios establecidos en los siglos XIX y XX, entre los países latinoamericanos, las naciones europeas y los Estados Unidos de América, observando las similitudes en la implantación de la forma escolar moderna. Por otro lado, es innegable la circulación de educadores entre Argentina y Brasil en el mismo período, contribuyendo de forma significativa para que ese análisis sea establecido, y, para que podamos pensar la educación dirigida al trabajo observando contextos más específicos. (Tadielo \& Soares, 2016)

En consecuencia, partimos de la premisa que las historias están conectadas, por lo tanto, pensar los modelos educacionales representados en las leyes, en los espacios de Brasil y Argentina, puede traernos informaciones relevantes cuanto a las ideologías presentes en la formulación de esos reglamentos, y de qué maneras esos pensamientos repercuten en la organización de las culturas y de los sistemas educativos.Todo eso nos ha llevado a considerar que solo trabajando juntos, podríamos producir respuestas, aunque provisionales, acerca de la educación profesional en esos dos países.

En este estudio, no pretendemos solamente explicitar y analizar separadamente la Educación Profesional en Brasil y Argentina en el recorte temporal propuesto, sino hacer un intento de aproximación de esas realidades, que juzgamos atender a las demandas particulares, y que dialogan en el contexto de la América Latina. Luego, este trabajo nace a partir de una sugestión de estudio comparado en el campo de la história de la educación profesional argentina y brasileña, tomando como principal material de análisis las fuentes documentales provenientes de decretos y leyes institucionales, que son: el Decreto Leoncio de Carvalho en 1879 en Brasil y los aspectos aproximativos con la Ley n.1.420, promulgada en Argentina en 1880; también analizando la Ley de Directrices y Bases de la educación brasileña ${ }^{\circ}$. 9.394/96, la Ley de Educación Nacional Argentina $n^{\circ}$. 26.206/06 y la Ley específica de $n^{\circ}$. 24.521/95, leyes educacionales desarrolladas en el transcurso del siglo XX en esos dos países. Citaremos también la implementación en 2006 del proyecto entre la Coordinación de Perfeccionamiento de Personal de Nivel Superior - CAPES y la Secretaría de Ciencia y Tecnología - SECyT2, que tenía por objetivo investigar de forma conjunta la constitución y las reformas de los sistemas educativos en Brasil y Argentina.

Entonces, para mejor enunciar, organizamos este análisis, partiendo inicialmente del estudio bibliográfico y del uso de las fuentes legales relativas a la Educación Profesional en Brasil y Argentina. Establecemos un panorama introductorio a través 
del análisis comparado de la educación, a partir de la perspectiva histórica, política y social de producción de estas leyes y cómo orientan las políticas de implementación dirigidas a la educación para el trabajo.(Vieira \& Souza Junior, 2016) A continuación, analizamos el contenido de estas leyes haciendo relaciones, aproximaciones y distanciamientos por medio de los argumentos expresos en esos reglamentos. Y, conjuntamente, observamos las influencias que los organismos internacionales ejercen en la configuración y en la implementación de esas políticas educacionales.

\section{Aspectos Teórico Metodológicos Sobre los Usos de las Fuentes en la Historia Comparada de la Educación}

Las leyes y decretos educacionales de Brasil y Argentina se constituyen como fuente historiográfica del presente análisis. Para ello, la identificación y la interpretación de esas fuentes se presentan como elementos constituyentes de esa investigación, conformándose como fundamental con respecto a investigar los modelos educacionales construidos en esos espacios, y sus influencias mutuas en el paso del siglo XIX al siglo XX, considerando el escenario de la escolarización moderna. Nos fijamos en el aspecto que las fuentes no hablan por sí mismas, sino que son vestigios y testimonios que responden a un número limitado de hechos históricos, trayendo interpretaciones a las preguntas que les son presentadas por la investigación. Por lo tanto, la fuente se entiende aquí como una construcción del investigador, y como tal, se le atribuye un sentido. (Ragazzini, 2001)

Para Ragazzini (2001) hay dos formas de discutir las fuentes en la ámbito de la educación, una considerando las fuentes de la escuela, es decir, aquellas que provienen directamente de las prácticas escolares, y, una segunda forma, que se refiere a las fuentes para la historia de la escuela y de la educación, que ayudan a interpretar y escribir esa historia. Es en esta segunda forma que vamos a detenernos, considerando que las prácticas regulatorias del estado, relacionadas a las leyes educacionales, aunque no sean directamente transpuestas para una práctica escolar en cuanto son lanzadas e instituidas políticamente, nos ayudan a interpretar las ideas en torno a las nociones de escuela en cada tiempo y espacio a que se destinan.

En la perspectiva de evaluar legislaciones como fuentes históricas que se adecuan a la producción de este trabajo, André Paulo Castanha (2011) señala que entre las muchas fuentes que pueden subsidiar las investigaciones históricoeducativas, se destaca la legislación educacional, debido al gran número de temas y cuestiones que están explícitos e implícitos en ella. Sin embargo, no basta simplemente acercarnos a las leyes de la educación para producir un estudio coherente y consistente. Es esencial encontrar una teoría y una metodología adecuada para su uso, de manera que las leyes puedan revelar, mucho más de lo que está prescrito en sus artículos y párrafos.

Nuestra investigación es de base cualitativa y hace uso del análisis textual discursivo como herramienta analítica, una vez que dicho análisis crea espacios de reconstrucción, comprensión de la producción y significados sobre los fenómenos estudiados. Según Moraes y Galiazzi, "el análisis textual discursivo es un abordaje de análisis de datos que transita entre dos formas consagradas de análisis en la investigación cualitativa, que son el análisis de contenido y el análisis de discurso". Sin embargo, podemos asignar un sentido específico a tal análisis, vislumbrando el abordaje que queremos ejecutar en nuestra investigación para la construcción de argumentos. "El análisis textual discursivo más de un conjunto de procedimientos definidos constituye metodología abierta, camino hacia un pensamiento investigativo [...]". (Moraes \& Galiazzi, 2006, pp. 118119)

En ese análisis, las realidades investigadas, examinadas, decodificadas, no se dan listas para ser descritas e interpretadas. Son inciertas e inestables mostrando que "las ideas y teorías no reflejan, sino que traducen la realidad" (Moraes \& Galiazzi, 2006, p. 121). Y, es en ese movimiento que los contenidos de las leyes y decretos son examinados y conectados, pues tratándose de una realidad Latinoamericana, más particularmente de Brasil y Argentina esos reglamentos, analizados bajo la égida del discurso, nos permite ver las aproximaciones y distanciamientos necesarios para comprender esas realidades 
múltiples.

Pensando en esas realidades conectadas, utilizamos los presupuestos de la Historia comparada de la educación, considerando las relaciones entre las leyes educacionales de Brasil y Argentina, comparándolos en su aspecto político-social, contexto de creación y alteraciones, así como la realización de los intercambios entre esos modelos educacionales de estos países. Y como afirma Saviani (2001):

En rigor, la historia comparada de la educación es algo que, en Brasil (y creo que eso vale para toda América Latina), todavía está por hacer. [...] En ambos casos no se trata, propiamente, de estudios comparados porque la comparación no es hecha, siendo dejada como una tarea atribuida a los lectores. (p.10).

Profundizando nuestro entendimiento en cuanto al uso de este método, Ciavatta (1992) nos trae un análisis con sesgo filosófico. Ella lo llama "comparativismo", la comparación hecha a través de una mirada cargada de un complejo de inferioridad, donde se busca reflejar en países extranjeros del capitalismo central como ideales a ser seguidos. Por lo tanto, es un movimiento de comparación que parte desde su principio, con tendencias de mantenimiento de distanciamientos, ya sea por la caminata histórica recorrida por países en contextos tan diversos, o por la influencia de organismos internacionales que se posicionan sutilmente, o con acciones incisivas y directas, con el fin de mantener el estatus de dependencia de los países del capitalismo periférico.

$\mathrm{Al}$ asumir esta posición de inferioridad, evita la posibilidad de comprenderse en conjunto con sus vecinos, reforzando un estereotipo de exotismo e incapacidad para superar las dificultades que se les imponen. Ciavatta (1992) trae como hipótesis, que la relación de dominación es establecida incluso en el dominio escolar, especialmente en lo que concierne a la planificación, metas a ser alcanzadas, estrategias y caminos a seguir, ambos establecidos y diseminados por organismos internacionales, incluso, interfiriendo en la mayor parte de los estudios comparados desarrollados hasta entonces.

En ese sentido, nuestra elección por un objeto de comparación más cercano, que comparte situaciones económicas, políticas y sociales similares, nos permite una mejor visión de conjunto sobre la educación implementada en esas naciones. Y, aún, nos permite percibir que proyectos, incluso del capital hegemónico, fueron impuestos para esas realidades. Sin embargo, es preciso considerar que al confluir investigaciones elaboradas en diferentes países se hace necesario tener cuidado para no tratar esas realidades distintas como si vivieran situaciones idénticas, en la medida en que los contextos particulares de las prácticas sociales también participan en las reconstrucciones históricas y deben ser considerados. Siendo así,

La comprensión de la distancia dramática que nos separa y separa nuestros países y nuestra educación de los países económicamente desarrollados es el primer punto a merecer reflexión. El segundo paso es la demarcación de los espacios históricos donde, por caminos diferentes, podemos movernos. En cierto sentido, unos y otros son sistemas incomparables, en la medida en que son fruto de procesos históricos particulares, de trayectorias singulares, no obstante sus similitudes. (Ciavatta, 1992, p.38)

Además, es preciso considerar que el proceso histórico se constituye por las interacciones entre esas realidades. Sería impropio, por ejemplo, entender que la educación brasileña y la educación argentina se hubieran desarrollado nacionalmente, bajo condiciones exclusivamente peculiares. Eso sería suponer que las sociedades pudieran llegar a formas educativas e instituciones políticas similares como una consecuencia natural del proceso histórico, y que las naciones caminen linealmente en dirección al progreso, sin considerar las influencias mutuas que pueden ocurrir entre espacios contiguos.

Por consiguiente, Ciavatta (1992) nos presenta que: "comparación es un proceso de percibir las diferencias y semejanzas y de asumir valores en esa relación de reconocimiento mutuo. Se trata de entender al otro a partir de él mismo y, por exclusión, si percibir en la diferencia" (p. 14). De ese modo, el acto de comparar sirve para "conocer a los otros, a nuestro interlocutor, a nuestro vecino, etc. y, en ese acto, profundizar el conocimiento de sí mismo y del otro". (Ciavatta, 1992, p.14) 


\section{Resultados y Discusiones: la Dualidad Presente en la Educación Profesional de Brasil y Argentina}

Se examina erróneamente que los sistemas educativos son objetos aislados, desprovistos de sus contenidos sociales, políticos y económicos. Sin embargo, entendemos que esos aspectos deben tenerse en cuenta. Una vez que se refieren a la dualidad presente en la educación brasileña, y que también se presentan de manera significativa en la realidad Argentina.

En la historia de la educación, una de sus marcas es la formación para el trabajo destinada a las clases populares. Fue así en Brasil desde las primeras experiencias de institucionalización de la enseñanza profesional, con la creación de los Colegios de Fábricas, en 1809, por D. João VI, destinados obligatoriamente a los huérfanos y desvalidos3. También fue así con la creación, en 1909 de las 19 Escuelas de Aprendices Artífices, destinada "a los hijos de los desfavorecidos de la fortuna", (Colombo, 2020) y aún con la creación de la Ley Orgánica de la Enseñanza Industrial, en 1942, y también con la creación del Sistema S4 que tenía la finalidad de ofrecer "una formación práctica a los jóvenes pobres". (Araújo, 2019)

Este movimiento se repite también en Argentina, donde se resalta la idea de que muchas reformas y políticas educativas, en el paso del siglo XIX al XX, pretendían "civilizar a los bárbaros", o sea, los pueblos nativos. Luego, predominaba en la organización de la educación el objetivo de educar a las élites, y, a través de una educación excluyente, selectiva y contradictoria, instruir "a los demás". (Vidal \& Escolani, 2009)

En el siglo XIX, ya se había desarrollado en Europa el fenómeno de la educación elemental de masa, proceso en construcción en los dos siglos anteriores, de formación de una nueva sociedad basada en la afirmación del individualismo, del universalismo y de la racionalidad, fenómeno relacionado con la formación de los Estados Nacionales (Ramirez \& Boli, 1987). Para este mismo recorte temporal, en la constitución de los Estados latinoamericanos, esa discusión se aclara en los discursos legislativos. Teniendo en cuenta que con la proclamación de la Independencia de Brasil (1822) y de la República en Argentina (1816), no sólo se crearon regímenes políticos diferentes, sino que también se ha demandado la creación de instancias legislativas específicas, todos estos controlados por las élites de cada país. (Pasche \& Cury, 2020)

La centralidad adquirida por la educación en los embates políticos latinoamericanos en el siglo XIX, lleva una importante característica de los procesos políticos y culturales en curso. Lo que esas naciones tenían en común era la posibilidad de construir el Estado y la Nación por medio de un esfuerzo civilizatorio, una política de identidad, pretendía garantizar, en los diversos regímenes políticos, sea en el Brasil Imperial, sea en la República Argentina, una cultura política con fuertes marcas autoritarias abrazadas por las élites.

La idea de incorporación de forma subalterna, de las capas populares de la República, en el caso de Argentina, o al Imperio, en el caso de Brasil, dio lugar a un conjunto de acciones, las cuales tuvieron en la inversión discursiva en la educación uno de sus puntos más fuertes. Y, las leyes tuvieron gran influencia en la conformación de ese vocabulario, que tenía como objetivo la construcción de una nación que mantuviera intactos los privilegios de un pequeño grupo social, en detrimento de la mayor parte de la población.

Pessoa et al (2021) expone que la relación entre educación y trabajo es constante en los discursos educativos, sin embargo, esa relación es traída de manera descontextualizada, y en la mayoría de las veces privilegiando el trabajo en detrimento de la educación, o lo contrario. Para los autores ese enfoque es consecuencia de una dualidad estructural presente en el sistema capitalista, que concibe una educación más simple y mecánica para las clases trabajadoras. Orlando Bif et al (2021) también nos trae esa perspectiva, señalando que la escuela unitaria del pensamiento gramsciano, es decir, que propone una formación integral, se opone a la dualidad de una formación general y profesional reducida, dirigida únicamente para los

\footnotetext{
${ }^{3}$ En Brasil el término "desvalidos" se refiere a los niños y jóvenes pobres, la mayoría de las veces, abandonados o sin parentela.

4 Término que define el conjunto de organizaciones de las entidades corporativas de Brasil dirigidas al entrenamiento profesional, asistencia social, consultoría, investigación y asistencia técnica, que además de tener su nombre iniciado con la letra S, tiene raíces comunes y características organizacionales similares. Fuente: Agencia del Senado de Brasil.
} 
designios del capitalismo.

En ese sentido, era necesario equilibrar esas capas populares: controlar, educar, instruir, civilizar. Eran ideas continuamente movilizadas en los más diferentes discursos que pretendían crear, en el horizonte de expectativas de nuestras jóvenes naciones, un futuro grandioso y fuerte, aunque eso tuviera que ser hecho aniquilando ora a los enemigos internos, ora a los enemigos externos, como muchas veces se hizo en el siglo XIX.

La composición de las Cámaras deliberativas y la formación jurídica de los nuevos parlamentarios fueron cuestiones prioritarias en la construcción de las naciones independientes, tanto en lo concerniente a las estrategias de control y ordenamiento social, como en la producción de la identidad nacional. Esas fueron estrategias trazadas por las naciones y que repercutían directamente en el hacer escolar y en las prácticas educativas establecidas en la sociedad. La educación se presentaba como un mecanismo de apoyo a los intereses de los sectores políticos. (Gvirtz et al., 2009) Por lo tanto, las instituciones escolares se han constituido en uno de los más importantes elementos de integración política y de cohesión social, materializando la transmisión de los valores de los grupos dirigentes hacia el establecimiento de un nuevo orden.

Esta relación entre la educación formal, la civilización y los requisitos de ciudadanía, como estrategias combinadas de homogeneización y de diferenciación cultural y social, para vencer la "barbarie", mantuvo el control de los posibles efectos políticos de la instrucción de las clases subalternas, en su casi totalidad, dirigido hacia el trabajo manual. Contribuyendo así con el mantenimiento de la hegemonía de los grupos dominantes, esto puede ser verificado claramente en las leyes y decretos del período. Tomamos como base el Decreto $n^{\circ}$. 7.247, de 1879, proclamado por Carlos Leoncio de Carvalho en Brasil y la Ley $\mathrm{n}^{\circ} .1 .420$, promulgada en Argentina en 1880. Las dos corresponden prácticamente al mismo período y llevan esas marcas ya mencionadas.

La Ley no. 1.420, promulgada en Argentina en 1884 estaba constituida por 82 artículos distribuidos en nueve capítulos y establecía en el artículo $2^{\circ}$ que "la instrucción primaria debería ser obligatoria, gratuita, gradual, y dada conforme a los preceptos de la higiene". Esos preceptos de higiene consideraban el saneamiento moral y social de esas clases llamadas subalternas, importantes para su escolarización, que generalmente eran destinadas a los oficios manuales y a la educación dirigida al trabajo. Mientras las clases más ricas podían dedicarse a las actividades más intelectuales, garantizando sus posiciones políticas en la gestión de la nación, integrando los cuadros dirigentes de las instituciones. Cuando trata sobre ese aspecto, Ciavatta nos provoca a reflexionar por medio de Matta (Matta, 1987) que:

[...], el objetivo de de "civilizar la barbarie", para fines de progreso económico y consolidación de los Estados nacionales, va a generar - en Argentina, con Domingos Faustino Sarmiento, y en Uruguay, con José Pedro Varela, a finales del siglo XIX - la organización de la educación popular.(Ciavatta, 1992, p.21)

Es importante observar que las acciones de Domingos Faustino Sarmiento y de José Pedro Varela tuvieron el mismo propósito sanitizante que la propuesta de las escuelas de aprendizaje de oficio de Brasil, en cuanto al adiestramiento social. (Ciavatta \& Reis, 2016) Así, para las élites de esas naciones, la construcción de esas identidades modernas, no sería resultado de un esfuerzo colectivo, sino una acción de los "hombres de bien", sobre los "otros", incivilizados: las mujeres, los pobres, los sin ilustración, los habitantes del campo, los indios, los negros, los esclavos, los niños abandonados. Pues, la educación, en el plan elitista, tenía la función de homogeneizar las diferenciaciones culturales, que pretendían borrar y muchas veces incluso eliminar las culturas de determinados sujetos sociales que molestaban.

En el Decreto $\mathrm{n}^{\circ}$. 7.247, de 1879, proclamado por Carlos Leoncio de Carvalho en Brasil, también en sus numerosos artículos eran considerados los aspectos higienistas, y establecidos diversos criterios para conformación de la civilización que se proponía a liberarse de la barbarie, controlando a los indeseables, los destinan a funciones útiles a la nación. Además, tratándose de una educación dirigida al trabajo, vemos en esa ley el establecimiento de escuelas profesionales, destinada al 
aprendizaje de oficios, instituciones esas, en su mayoría dirigidas a las clases trabajadoras. Podemos conferir en el artículo noveno del Decreto:

Art. $9^{\circ}$. Crear o auxiliar en el municipio de la Corte y en los más importantes de las provincias escuelas profesionales, y escuelas especiales y de aprendizaje, destinadas, las primeras a dar la instrucción técnica que más interese a las industrias dominantes o que convenga crear y desarrollar, y las segundas a la enseñanza práctica de las artes y oficios de más inmediato beneficio para la población y para el Estado, conforme las necesidades y condiciones de las localidades. (Decreto ${ }^{\circ} 7.247,1879$ )

Se ha atribuido a las personas, dependiendo de su origen social, la clasificación en "órdenes superiores" o de las "clases inferiores". El carácter concreto del pensamiento de los órdenes inferiores contra las cualidades intelectuales, verbales y abstractas del pensamiento de la clase alta. Con base en esas dicotomías, o incluso dualidades, se establecía un eslabón clasificador de los saberes: "para eso, tanto en la sociedad como en el cuerpo, la cabeza era reflectiva, manipuladora, controladora; la mano, irrefletida, mecánica, determinada por instrucciones". (Goodson, 2018, p. 105) La instrucción mecánica, técnica estaría dirigida a las clases populares, en el sentido de volverse útiles a la nación, y responder a los intereses de las "industrias dominantes", como se destacó en el Decreto de Leoncio de Carvalho (1879).

En el ochocientos es importante destacar que el campo de lo jurídico y de lo educativo aún se estaba configurando, lo que hacía las fronteras más fluidas. Por eso, las fuentes legislativas son muy relevantes para la historia de la educación, teniendo en cuenta que esos campos se confundían, y las acciones educativas tenían que pasar la criba de las estrategias políticas, pensadas y articuladas por intereses específicos de las clases dominantes.

En el análisis de las leyes y utilizando procedimientos relacionales, podemos percibir la circularidad de los sujetos educacionales, modelos pedagógicos circunscritos en la legislación y objetos didácticos, las relaciones internacionales establecidas entre países, y las apropiaciones efectuadas en múltiples escalas. Esas historias interconectadas ofrecen contribuciones a la comprensión de la dimensión de la práctica social, amparando las preocupaciones historiográficas de una historia comparada (Barros, 2019). Es importante también notar que existen alcances y límites de tal proceso de comparación, al observar contextos específicos, sin embargo, las aproximaciones son provechosas.

Brasil y Argentina representaban modelos en la frontera sur de las Américas, con eso, era común las aproximaciones ejecutadas por figuras políticas, en el caso aquí explicitado, las comparaciones sobre los modelos educacionales, tomando como ejemplo José Ricardo Pires de Almeida, que publica al final del período monárquico de Brasil en 1889, el libro " $\mathrm{L}$ ' Instruction publique au Brésil: Historie et Legislation (1500-1889)". En esa obra, el autor hacía un elogio a la educación pública del Imperio. Se decía obligado al "deber y casi misión de restablecer la verdad". "Brasil es, ciertamente, de entre todos los países de América del Sur, aquel que mayores pruebas ha dado de amor al progreso y a la civilización". La principal referencia de este autor era Argentina. No solo pretendía afirmar el liderazgo brasileño en términos educativos, sino destacar la superioridad del régimen monárquico sobre el republicano. (Gvirtz, et al., 2009)

En conformidad con esa perspectiva, bajo las palabras de Ruy Barbosa, figura importante de la política brasileña, Argentina se presentaba como un modelo a seguir. Mientras José Ricardo Pires de Almeida, exaltaba los hechos del Brasil monárquico y escribía después de haber sido sancionada la Lei $\mathrm{n}^{\circ}$. 1.420, en 1884 en Argentina. Ruy también elaboró un extenso dictamen sobre el Decreto $n^{\circ}$. 7.247, promulgado en 1879 en Brasil. "Tomar esos dos marcos legislativos como objeto de estudio nos parece una estrategia relevante para entender las reformas educativas en los dos países como prácticas sociales, consideradas a partir de una historia social, cultural y pedagógica". (Gvirtzet, al., 2009, p. 34).

En el caso argentino, la ley 1.420 de "Educación Comùn", sancionada en 1884 y cuyos principales antecedentes fueron la ley Ferry en Francia y el Congreso Pedagógico Nacional de 1882, fue un marco en cuanto al modelo 
formador asumido por Argentina para aquel momento y para las generaciones futuras. Regulaba la educación y fijaba la obligatoriedad para los niños de 6 a 14 años, la gratuidad y laicidad de la enseñanza, determinando los contenidos básicos de la instrucción. El Estado tenía el deber de proveer las escuelas necesarias para garantizar la atención de toda la población en edad escolar. (Gvirtz, et al., 2009, p. 34).

La instrucción pública asumida por el Estado, en nuestro caso estudiado de Argentina y Brasil, también estaba regida por leyes de mercado, que profesaban un discurso que podría "practicar una acción educativa característicamente protectora, garantizadora, amplia, sistematizadora y múltiple". Lo que en realidad no era ejecutado, lo que ocurría era una acción muy dirigida hacia intereses específicos de mantenimiento del status quo. Vale recordar que el debate se daba después de la promulgación de la Ley del Vientre Libre, en 1871, poniendo en la agenda política la necesidad urgente de definir la acción del Estado en lo que vislumbraba a la formación del pueblo brasileño contemporáneo, y las generaciones futuras en la inminencia de la incorporación de las masas esclavas a la población nacional. La concepción de Estado educador, emergente bajo la pena de Ruy Barbosa, encontraba eco en la legislación promulgada en Argentina en 1884. (Gvirtz, et al., 2009)

Otro aspecto relevante entre esas dos naciones, que también se transformó en política de estado y consecuentemente, se hacía presente en la legislación, fue el concerniente a laicidad de la enseñanza y el Estado Liberal, eso ya considerando finales del siglo XIX y el transcurrir del siglo XX. En Argentina, la expropiación de la iniciativa educativa de la Iglesia venía acompañada de una legislación que hacía del Estado el principal agente formador de la infancia y de la juventud. En Brasil, los debates en torno a la educación obligatoria se revestían, además de la laicidad, de consideraciones acerca de la recomposición social y política, proveniente de la previsible liberación de los esclavos a ocurrir en 1888. (Chalhoub, 2016) La proclamación de la República en Brasil en 1889 reforzaría los conflictos sobre la problemática, aunque las acciones resultantes no llegaran a consolidar la centralización estatal en materia educativa. Al contrario, las primeras décadas republicanas consagraron la división de la competencia administrativa sobre la enseñanza elemental, instituida desde 1834, observando un modelo federativo en lo que respecta a la instrucción pública.

Además de los aspectos ya mencionados sobre el siglo XIX, y las aproximaciones en términos de la construcción de las identidades nacionales tanto en Brasil, como en Argentina, cuando pasamos para análisis comparativo en el transcurrir del siglo XX, alcanzando el inicio del siglo XXI, También encontramos aproximaciones que refutan percibir esas realidades de manera aislada. Pensando en esas temáticas conectadas, la Coordinación de Perfeccionamiento de Personal de Nivel Superior de Brasil - CAPES y la Secretaría de Ciencia y Tecnología - SECyT, desarrollaron a partir del año 2007, a través de la Universidad de São Paulo - USP un proyecto con el propósito de investigar de forma conjunta la constitución y las reformas de los sistemas educativos de forma simultánea en Brasil y Argentina. La premisa establecida es que las historias de esas dos naciones están interconectadas y que solo trabajando juntas sería posible producir respuestas sobre los sistemas educativos de esos dos países.

Al visitar las primeras décadas del siglo XX, es importante destacar que a nivel legislativo no había una homogeneización de la escuela primaria en Brasil. Las numerosas reformas educativas establecidas en las primeras décadas del siglo XX, a pesar de compartir los principios de la Escola Nova5, operaron cambios curriculares cada una a su modo. Incluso con la creación del Ministerio de la Educación y Salud en 1930, ese escenario de descentralización no fue modificado. Solamente en 1946, es que serían promulgadas las Leyes Orgánicas de la Enseñanza Primaria y Normal, antecedidas por las leyes referentes a la enseñanza profesional, promulgadas aún en el gobierno de Getúlio Vargas en 1942.

Analizando el caso argentino, entre 1976 y 1983, ocurrió el golpe militar, lo que causó una de las páginas más sangrientas de la historia argentina. En esos años, la dictadura avanzó en la destrucción del sistema educativo, particularmente

${ }^{5}$ La expresión Escola Nova en el panorama educativo ya era utilizada en el período de la Primera República en Brasil, sin embargo, solamente a finales de los años 1920, con la reforma de Fernando de Azevedo en Río de Janeiro, el término pasó a significar los esfuerzos de renovación del sistema escolar y de ruptura simbólica con las antiguas estructuras educativas.(Vidal, 2013, p. 582) 
de la enseñanza pública, a través del estímulo a la iniciativa privada, municipalización de las instituciones escolares, represión a la Comunidad escolar por medio de persecuciones e incluso asesinatos de profesores y alumnos, y, la restauración de valores católicos, conservadores y tradicionales. En cuanto a la educación profesional, se percibe en ese período un incremento de esas iniciativas, con vistas a que los sectores privados, muy próximos a los sectores dirigentes de la dictadura, veían con muy buenos ojos la simplificación del contenido propedéutico para garantizar un mayor incremento de la cualificación profesional, para formación de mano de obra poco especializada. Eso se ajusta al carácter estrictamente técnico e instrumental incentivado en ese período por el tecnicismo educacional, en el cual la sociedad debería amoldarse a las demandas industriales y tecnológicas, atendiendo al sistema capitalista.

En Brasil, el escenario a partir del golpe civil militar de 1964 a 1985 no fue diferente de Argentina, es preciso resaltar que en esos dos países la actuación de los organismos internacionales, en ese caso en específico, de instituciones estadounidenses, el apoyo y la financiación de las dictaduras en toda América Latina, tenía por objetivo garantizar la permanencia de la posición subalterna de esos países, y, garantizar una masa de trabajadores controlados y superficialmente instrumentalizados para atender sus demandas del mercado internacional. En los veinte años de dictadura en Brasil, la educación presenció con la Ley $\mathrm{n}^{\circ}$. 5.692, de 1971, la ampliación de la obligatoriedad escolar para ocho años de estudio, la expansión del número de escuelas en funcionamiento y, al mismo tiempo, la ambigua relación con la formación del trabajador por la imposición de una formación profesional en nivel técnico. (Gvirtz, et al., 2009) Esta ambigüedad se contrapone al aumento del tiempo de escolarización, ya que se observa la baja calidad de la enseñanza, el aumento de alumnos en clase, el descenso de los salarios de los profesores, la instalación de las escuelas en edificios precarios.

Cuando analizamos el carácter capitalista mercantil presente en la educación y su combinación entre estudio y trabajo, así como trabajo manual/trabajo intelectual, percibimos que están presentes en todos los niveles escolares. Lo que difiere es la cualificación profesional y técnica en relación al estudio y trabajo con vistas a la atención de las necesidades de la industria, bajo la fuerte presencia de los empresarios y de los intereses capitalistas de formación de mano de obra. En ese sentido, los años 1980, los vientos de la redemocratización, la nueva Constitución y la LDB en trámite en el Congreso Nacional brasileño en esa década, posibilitó la reanudación de la defensa de la educación politécnica. (Ciavatta, 1992) A pesar del ideal de la educación politécnica, el referencial de la educación profesional no ha abandonado el carácter dual de la formación, en el contexto en el que la mayoría de los estudiantes pertenecen a las capas populares y, principalmente, para ellos continuó siendo destinada una educación vinculada a la formación profesional, teniendo en vista la atención a las exigencias del neoliberalismo. (Almeida et al., 2016)

Las reformas educativas de los años noventa en Argentina y Brasil priorizaron el "conocimiento" como un factor estratégico de singular relevancia para el logro de la competitividad y el desarrollo. La capacidad de incorporar conocimientos técnicos y nuevas tecnologías al sistema productivo impulsó la reorganización de los sistemas educativo y escolar.

Necesitamos considerar también la actuación de los organismos internacionales en el campo educativo en ese período, que está enraizada en las cuestiones administrativas de planificación y gestión de la enseñanza, en especial de los países clasificados de economía emergente. Las políticas que estos organismos defienden parten de una premisa de privatizaciones, eximiendo al Estado de su responsabilidad gerencial. Esas reformas educativas, principalmente en los años 1990, cumplieron las directrices del Banco Mundial, en una política de resultados que implica en la desvalorización y en la mecanización de la enseñanza.

Santos e Tavares (2020), analizan que organismos internacionales actuaron en la educación en los años 90 expropiando los derechos de las naciones en desarrollo de orientar los horizontes filosóficos, materiales y técnicos de sus políticas educativas en contrapartida a los préstamos concedidos por esas instituciones. El discurso empleado era la preparación de una mano de obra necesaria. [...] "El pensamiento hegemónico capitalista impacta en la educación profesional 
por medio de currículos mínimos y reduccionistas, comprometidos esencialmente con la adquisición de competencias técnicas y unilaterales para clase trabajadora" (p. 4).

Ese proyecto educativo de descentralización de la responsabilidad del Estado con la educación fue organizado para que los organismos internacionales bajo la lógica del modelo liberal y de la gestión gerencial asumieran cada vez más las responsabilidades en lo que se refiere a la educación. La educación se incorpora al sector de servicios y se gestiona como una actividad lucrativa en el campo de los negocios, que necesitaría de mano de obra cualificada y alineada a sus objetivos de ajustes políticos, económicos y sociales. "El nuevo paradigma, orientado a la productividad del mercado, demandaba requisitos diferenciados de educación general y cualificación profesional de los trabajadores para atender a las nuevas demandas" (Shiroma et al., 2007).

A pesar de la difusión de estos principios, en 2006 se aprobó en Argentina una nueva Ley de Educación Nacional (LEN), que destaca la necesidad de que el Estado Nacional tenga un papel activo, garantizando el acceso y permanencia en la escuela y combatiendo la reproducción de las desigualdades de origen social. Sin embargo, la ley tiene algunas debilidades, como la ausencia de tiempos máximos establecidos para el cumplimiento de los principales objetivos, la universalización de la educación infantil y la educación en tiempo completo.

En el cuadro abajo ponemos lado a lado las dos principales leyes educativas que más contemporáneamente modificaron los sistemas educativos de ambos países, en él presentamos datos numéricos que colaboran para comprender mejor los caminos trazados en cada país en cuanto a la oferta de educación profesional, que nos posibilitan hacer aproximaciones o distanciamientos entre las legislaciones de los dos países:

Tabla 1. Cuadro comparativo con aspectos generales de las Leyes de Educación brasileña (LDB) y argentina (LEN).

\begin{tabular}{|c|c|c|}
\hline & $\begin{array}{l}\text { Ley de Directrices y Bases de la } \\
\text { Educación (LDB) }\end{array}$ & $\begin{array}{l}\text { Ley de Educación Nacional de } \\
\text { Argentina (LEN) }\end{array}$ \\
\hline Promulgada en: & 20/12/1996 & $27 / 12 / 2006$ \\
\hline Número de la Ley: & 9.394 & 26.206 \\
\hline Número de artículos: & 92 & 145 \\
\hline Presenta Metas a alcanzar: & $\begin{array}{c}\text { No. Están en el Plan Nacional de } \\
\text { Educación }\end{array}$ & Sí \\
\hline Tiempo para Promulgación: & 8 años & menos de 1 mes \\
\hline Origen Legislativa: & Iniciativa Parlamentaria & Poder Ejecutivo \\
\hline $\begin{array}{l}\text { Construcción con participación } \\
\text { social }\end{array}$ & Sí & Sí \\
\hline $\begin{array}{l}\text { Propone un sistema unificado, } \\
\text { articulado, universal y nacional }\end{array}$ & Sí & Sí \\
\hline $\begin{array}{c}\text { Prevé Recursos para la Educación } \\
\text { Privada }\end{array}$ & Sí. Pero es bastante restrictivo. & Sí. Siendo más amplio. \\
\hline $\begin{array}{l}\text { Delimita y evalúa la actuación del } \\
\text { sector privado }\end{array}$ & Sí & Sí \\
\hline $\begin{array}{l}\text { Número de artículos que versan } \\
\text { sobre la Educación Superior }\end{array}$ & 15 & $\begin{array}{l}\text { Solo 4. Pero se detalla en ley específica } \\
\qquad\left(\text { Ley }^{\circ} 24.521\right)\end{array}$ \\
\hline $\begin{array}{l}\text { Número de artículos sobre la } \\
\text { educación profesional }\end{array}$ & 4 & 1 \\
\hline
\end{tabular}

Fuente: Elaboración propia, a partir de las discusiones en: (Castro, 2007). 
Ambas legislaciones presentadas en la tabla I destacan la importancia de asegurar educación de calidad, con equidad, visando la formación ciudadana, con respecto a la diversidad, la ética y el compromiso social. Sin embargo, la legislación argentina propone más explícitamente una integración interinstitucional, especialmente entre los órganos directamente relacionados con la educación, la ciencia y la tecnología.

La educación técnico-profesional argentina, como se muestra en la tabla, tiene un artículo y es regida por ley específica, tiene características más restringidas que la educación profesional brasileña, una vez que aquella se vincula necesariamente a la educación secundaria o superior. Vemos a continuación el fragmento de la ley relacionada con la educación profesional en Argentina:

Art. $38^{\circ}$. La Educación Técnico Profesional es la modalidad de la Educación Secundaria y la Educación Superior responsable de la formación de técnicos medios y técnicos superiores en áreas ocupacionales específicas y de la formación profesional. La Educación Técnico Profesional se rige por las disposiciones de la Ley $\mathrm{N}^{\mathrm{o}} 26.058$, en concordancia con los principios, fines y objetivos de la presente ley. Esta modalidad se implementa en las instituciones de gestión estatal o privada que cumplen con las disposiciones de la Ley $\mathrm{N}^{\circ}$ 26.058. (Ley $\mathrm{n}^{\circ}$ $26.206 / 2006)$

Mientras que la LDB (Ley de Directrices y bases de la Educación Brasileña) admite esa articulación, pero también el desarrollo de diferentes estrategias de educación continua, en instituciones especializadas o en el ambiente de trabajo. Como observamos a continuación, la ley brasileña inserta la educación profesional en diversos campos y espacios de enseñanza y presenta en su legislación un panorama más completo de esa modalidad de educación: o Art. 39. "La educación profesional y tecnológica, en el cumplimiento de los objetivos de la educación nacional, se integra a los diferentes niveles y modalidades de educación y a las dimensiones del trabajo, de la ciencia y de la tecnología”. (Lei no 9.394 de 20 / 1996)

Esas dos naciones latinoamericanas se acercan, pero también poseen singularidades en cuanto a sus modelos educativos. Debido a su mayor alineamiento con las políticas de consolidación de los Estados nacionales inspiradas en el modelo e influencia europeas y, por consiguiente, al uso de la educación como medio de elevar la escolarización para infundir mejor un nuevo pensamiento nacional, el gobierno central argentino resistió la fuerte presión autonomista de las provincias y asumió diversas responsabilidades en materia educativa. Su sistema educativo, a pesar de conformarse de manera homogénea, no resistió a los procesos de descentralización que se han desatado en las últimas décadas, bajo el patrocinio de organismos internacionales como la UNESCO y el Banco Mundial (BIRD).

Sin embargo, la nueva Ley de Educación Nacional argentina revisa la línea de descentralización adoptada en el país en las últimas décadas, al atribuir al gobierno nacional prerrogativas con alcance regional ampliado, y estimular la mejora general de la calidad de los servicios educativos ofrecidos por el poder público. El impacto de esos movimientos descentralizadores tuvo poco efecto en Brasil, donde la gestión y el financiamiento de las escuelas de las primeras etapas de enseñanza ya eran históricamente descentralizadas por medio del movimiento de municipalización. Estructura criticada por nombres relevantes de la educación brasileña, como Anísio Teixeira, ya que en lugar de representar una mayor autonomía y un mayor potencial de desarrollo local, termina reflejando el abandono o la insuficiencia de los mecanismos impulsores del cambio.

\section{Conclusiones}

Ese escenario de reformas educacionales de carácter dual tanto en Brasil como en Argentina, son fuertemente direccionados por los intereses de las élites y de los organismos internacionales, y atravesados por caminos contradictorios en el proceso de construcción de las leyes, que podrán señalar para avances sociales, pero abren caminos para flexibilizaciones legales que amplían la capacidad de interferencias con profundo carácter mercadológico. Eso se refleja en una educación que 
tiene como objetivo principal la formación para el trabajo y no una formación humana integral.

Lamentablemente, muchas leyes y reformas no ofrecen necesariamente un cambio hacia el avance de la educación, no siempre son sinónimos de progreso e inclusión, considerando que muchas de ellas se ejecutan para favorecer y legitimar a los grupos que históricamente están en el poder, y que dirigen la construcción de los diversos artículos hacia una modernización sin cambios sociales.

Percibimos que los reglamentos educacionales, construidos en Brasil y en Argentina, en la segunda mitad del siglo XIX y en el transcurrir del siglo XX, presentan considerables semejanzas. Aunque, es necesario considerar, que cuando se reduce la escala de interpretación, surgen significativas diferencias que apuntan a modos particulares en cada uno de los países. Asimismo, defendemos el reconocimiento de la importancia histórica de las relaciones internacionales en la constitución de las dinámicas nacionales y en la organización de los sistemas de enseñanza en el contexto del desarrollo del capitalismo monopolista y sus fuertes influencias en América Latina.

Teniendo en cuenta los trabajos que seguían un modelo eurocéntrico de observación, elegimos enfocar nuestro análisis en América Latina y sus modelos particulares de construcción política, social y económica. En ese recorrido, se establecieron relaciones de contigüidad, rechazando el aislamiento de esas sociedades y culturas y enfatizando sus singularidades en el campo de la educación y de la educación profesional.

Considerando ese contexto, proponemos caminos que contraríen esos proyectos elitistas y liberales, buscando un proyecto de educación profesional transformador basado en los conceptos de emancipación y autonomía y que pretenda contribuir socialmente para el desarrollo de una sociedad incluyente. La propuesta de pensar esas naciones latinoamericanas en conjunto, que son consideradas economías periféricas, nos abre puertas para reflexionar sobre nuestro contexto con sus particularidades, pero también con sus muchas similitudes. Proponemos ese panorama inicial entre Brasil y Argentina, sin embargo, la educación profesional es una realidad educativa que atraviesa toda la Latinoamérica, y investigar esa relación con los demás países de ese recorte regional, cultural y político también forma parte de nuestras aspiraciones de investigaciones futuras, como también queda como propuesta para los demás investigadores, teniendo en cuenta que el conocimiento sobre nuestros vecinos, como ya nos alertaba Ciavatta (1992) puede profundizar el conocimiento sobre nosotros mismos y sobre el otro.

\section{Referencias}

Barros, J. D’A. (2019). Fontes Históricas - uma introdução aos seus usos historiográficos. [Fuentes Históricas: una introducción a sus usos historiográficos.] Editorial Vozes.

Castro, M. L. O. de. (2007). Brasil e Argentina: Estudo Comparativo Das Respectivas Leis Gerais Sobre Educação. [Brasil y Argentina: Estudio Comparativo De las Respectivas Leyes Generales Sobre Educación.] Consultoria Legislativa do Senado Federal. COORDENAÇÃO DE ESTUDOS. Brasília:Textos para discussão 32 .

Chalhoub, S. y P., \& Magalhães, A. F. (2016). Pensadores negros - pensadoras negras; Brasil, séculos XIX e XX. [Pensadores negros - pensadoras negras; Brasil, siglos XIX y XX.] Rio de Janeiro; Belo Horizonte: MC\&G Editorial; Editorial Fino Traço

Ciavatta, M. (1992a). Estudos comparados em educação na América Latina. [Estudios comparados en educación en América Latina.] Livros do Tatu/Cortez.

Ciavatta, M., \& Botelho, J., dos Santos, J. R., Cariello, L., Lima, M. Rocha, M., Reis, R., Rosa, R. Morais, S., Ferreira, S. N. (2019). A historiografia em trabalho-educação: como se escreve a história da educação profissional. [La historiografía en trabajo-educación: cómo se escribe la historia de la educación profesional.] Uberlândia: Navegando Publicações.

Cunha, L. A. (2005). O ensino de ofícios artesanais e manufatureiros no Brasil escravocrata. [La enseñanza de oficios artesanales y manufactureros en Brasil esclavista.] Flacso; Editorial UNESP.

Gondra, J. G. \& Schueler, A. (2008). Educação, poder e sociedade no Império Brasileiro. Cortez.

Goodson, I. F. (2018). Currículo: Teoria e História. Coleção Ciências Sociais da Educação. (15a ed.), Vozes.

Kuhlman Júnior, M. (2015). Infância e Educação Infantil: uma abordagem histórica. (7a ed.), Mediação.

Shiroma, E. O., \& Moraes, M. C. M., Evangelista, O. (2007). Política Educacional. (4a ed.), Lamparina. 
Vidal, D. G. \& Ascolani, A. (org.). (2009). Reformas educativas no Brasil e na Argentina: ensaios de história comparada (1820-2000). [Reformas educativas en Brasil y Argentina: ensayos de historia comparada (1820-2000).] Cortez.

de la Fuente. Al., \& Andrews, G. R., Ferreira, R., Seijas, T., Wade, P., Fischer, B., Grinberg, K., Mattos, H., Guridy, F., Hooker, J., Paschel, T., Alberto, P., Hoffnung-Garskof, J., Sommer, D., Moore, R., Johnson, P. C., Palmié, S., Offen. K, Putnam, L., Jones, J. (2018). coordinación general de George Reid Andrews. Estudos afro-latino-americanos: uma introdução. En: Desigualdade: Raça, Classe e Gênero. 1a ed. - Ciudad Autónoma de Buenos Aires: CLACSO.

Gvirtz, S., \& Vidal, D. G., Biccas, M. de S. (2009). As reformas educativas como objeto de pesquisa em história comparada da educação na Argentina e no Brasil. En Reformas educativas no Brasil e na Argentina: ensaios de história comparada da educação (1820-2000). São Paulo: Cortez.

Kuenzer, A. Z., \& Machado, L. R. S. (1982). A pedagogia tecnicista. En: Mello, G. N. (org.) Escola nova, tecnicismo e educação compensatória. São Paulo: Loyola.

Matta, R. da. (1987). Relativizando. Uma Introdução à Antropologia Social. (pp. 86 - 118). Rocco.

Almeida, V., \&Bortoloto, C., Lopes C. B. (2016). Ensino Médio: trajetória histórica e a dualidade educacional presente nas diferentes reformas. Revista Perspectiva, 34(2), 555-581. 10.5007/2175-795X.2016v34n2p555

Araújo, R. M. L. (2019). Sobre dualidade, desigualdade e diferença. Ensino médio brasileiro: dualidade, diferenciação escolar e reprodução das desigualdades sociais. Cadernos de Pesquisa, (4). 9-52. 10.18764/2178-2229.

Barros, J. D’A. (2019). Histórias interconectadas, histórias cruzadas, abordagens transnacionais e outras histórias. Secuencia. 103, e1528. $10.18234 /$ secuencia.v0i103.1528.

Bif, O., \& Maldaner, J. J., Rythowem M., Pasqualli, R. (2021). Educação e trabalho no país da mais valia: a EPT no contexto do movimento do capital no Brasil. Research, Society and Development, 10 (10), 1-14. 10.33448/rsd-v10i10.19033

Castanha, A. P. (2011). O uso da legislação educacional como fonte: orientações a partir do marxismo. Revista HistedBR on-line, (41), e309-331. 10.20396/rho.v11i41e.8639912

Ciavatta, M., \& Reis, R. (2016). O passado escravista no presente: a sociologia histórica de Luiz Antônio Cunha. Revista HistedBR on-Line, (70), 70- 86. 10.20396/rho.v16i70.8649209

Colombo, I. M. (2020). Escola de Aprendizes Artífices ou Escola de Aprendizes e Artífices? Educar em Revista, e71886. 10.1590/0104-4060.71886

Moraes, R., \& Galiazzi, M. do C. (2006). Análise textual discursiva: processo reconstrutivo de múltiplas faces. Ciência \& Educação (1), 117-128. $10.1590 / \mathrm{S} 1516-73132006000100009$

Pasche, A. M. L., \& Cury, C. E. (2020). Independências políticas na América sob a perspectiva da História da Educação. SciELO em Perspectiva: Humanas. https://humanas.blog.scielo.org/blog/2020/12/03/independencias-politicas-na-america-sob-a-perspectiva-da-historia-da-educacao/

Pessoa, A. R., Gimezez, J., Silva, M. A. da., Ormond, N. F. P., Genuíno, N., Rocha, R.F.S. de S., Rocha, P. C. da S. (2021). Trabalho como princípio educativo na Perspectiva da Educação Profissional e Tecnológica. Research, Society and Development, 10 (15), 1-7. 10.33448/rsd-v10i15.22614

Ragazzini, D. (2001). Para quem e o que testemunham as fontes da história da educação? Educar em Revista, Editora da UFPR. (18), 13-28. 10.1590/01044060.231

Ramirez, F., \& Boli, J. (1987). The Political Construction of Mass Schooling: European Origins and Worldwide Institutionalization. Sociology of Education, $60(1), 2-17$.

Santos, L. F. dos \& Tavares, A. M. B. do N. (2020). Políticas para a educação profissional e a influência de organismos internacionais: pontos e contrapontos sobre a educação para trabalhadores no Brasil. Research, Society and Development, 9(7), 1-16. 10.33448/rsd-v9i7.4061

Saviani, D. (2001). História comparada da educação: algumas aproximações. 5(10), 5-16. Revista História da Educação.

Tadielo Cezar, T., \& Soares Ferreira, L. (2016). A relação entre educação e trabalho: um contexto de contradições e a aproximação com a educação profissional. Revista Ibero-Americana de Estudos em Educação, (4), 2141-2158. 10.21723/riaee.v11.n4.8248

Vidal, D. (2013). 80 anos do Manifesto dos Pioneiros da Educação Nova: questões para debate. Educação e Pesquisa, 39(3), 577-588. 10.1590/S151797022013005000007

Vieira, A. M. D. P., \& Souza Junior, A. de. (2016). A Educação Profissional no Brasil. Revista Interacções, (40), 152-169. 10.25755/int.10691

Coleção de Leis do Império do Brasil (1879). Página 196 Vol. 1 pt. II (Publicação Original). Legislação Informatizada - Decreto n. 7.247 , de 19 de abril de 1879. https://www2.camara.leg.br/legin/fed/decret/1824-1899/decreto-7247-19-abril-1879-547933-publicacaooriginal-62862-pe.html

Universidad Nacional de Córdoba Campus Virtual - UNC (2021). La SECyT. https://www.unc.edu.ar/ciencia-y-tecnolog\%C3\%ADa/autoridades.

Legislação Informatizada. Lei $N^{\circ}$ 9.394, de 20 de Dezembro de 1996. Publicação Original. https://www2.camara.leg.br/legin/fed/lei/1996/lei-9394-20dezembro-1996-362578-publicacaooriginal-1-pl.html

Leis da educação profissional e tecnológica no Brasil. http://portal.mec.gov.br/sesu-secretaria-de-educacao-superior/30000-uncategorised/67731-leislegislacao-e-atos-normativos-setec

Ley $\mathrm{n}^{\circ} 26.206$ ley de educación nacional, título I - disposiciones generales - capítulo I - principios, derechos y garantías. https://www.argentina.gob.ar/sites/default/files/ley-de-educ-nac-58ac89392ea4c.pdf 